\title{
Possible predisposition for colorectal carcinogenesis due to altered gene expressions in normal appearing mucosa from patients with colorectal neoplasia
}

Christian Hunnicke Petersen ${ }^{*}$, Badar Mahmood ${ }^{1}$, Christoffer Badsted ${ }^{2}$, Tina Dahlby², Hanne Borger Rasmussen², Mark Berner Hansen ${ }^{1}$ and Niels Bindslev ${ }^{2}$

\begin{abstract}
Background: Investigations of colorectal carcinogenesis have mainly focused on examining neoplastic tissue. With our aim of identifying potentially cancer-predisposing molecular compositions, we chose a different approach by examining endoscopically normal appearing colonic mucosa of patients with and without colorectal neoplasia (CRN). Directed by this focus, we selected 18 genes that were previously found with altered expression in colorectal cancer affected mucosa.

Methods: Biopsies of colonic mucosa were sampled from 27 patients referred for colonoscopy on suspicion of colorectal disease. Of these, 14 patients had present or previous CRN and the remaining 13 patients served as controls. Using qPCR and Western blot technique, we investigated mRNA and protein expressions. Expressions were investigated for selected kinases in the extracellular signal-regulated kinase/mitogen activated protein kinase (ERK/MAPK), the phosphoinositide 3-kinase/Akt, and the Wnt/B-catenin pathways as well as for selected phosphatases and several entities associated with prostaglandin $\mathrm{E} 2\left(\mathrm{PGE}_{2}\right)$ signaling. Colonic mucosal contents of $\mathrm{PGE}_{2}$ and $\mathrm{PGE}_{2}$ metabolites were determined by use of ELISA.

Results: We found up-regulation of ERK1, ERK2, Akt1, Akt2, PLA2G4A, prostanoid receptor EP3 and phosphatase scaffold subunit PPP2R1B mRNA expression in normal appearing colonic mucosa of CRN patients compared to controls.

Conclusion: Present study supports that even normal appearing mucosa of CRN patients differs from that of non-CRN patients at a molecular level. Especially expression of ERK1 mRNA was increased ( $p=0.007)$ in CRN group. ERK1 may therefore be considered a potential candidate gene as predictive biomarker for developing CRN. Further validation in larger cohorts are required to determine such predictive use in translational medicine and clinics.
\end{abstract}

Keywords: Extracellular signal-regulated kinase (ERK), Akt, Prostaglandin E2, $\beta$-Catenin, Colorectal cancer

\section{Background}

Investigating endoscopically normal appearing mucosa

Most investigations of tumorigenesis focus on alterations in neoplastic tissues or transformed cells in culture. Due to progressive derangement in neoplastic tissue of the genome, growth factor-signaling, and metabolic networks, screening of colorectal neoplasia (CRN) tissue

\footnotetext{
* Correspondence: christian1990hp@gmail.com

'Digestive Disease Center K, Bispebjerg Hospital, DK-2400 Copenhagen, Denmark

Full list of author information is available at the end of the article
}

itself demonstrates many alterations. Thus, investigations on CRN tissues provide limited information regarding possible predisposing factors and their role in transforming normal appearing mucosa to CRN. Screening of endoscopically normal appearing mucosa from patients with $\mathrm{CRN}$ and comparing it to mucosa of non-CRN patients may generate important new insights into identifying subjects with increased risk of developing CRN (risk stratification, predictive biomarker). As such, we and a few others have communicated altered expression of several

(c) The Author(s). 2019 Open Access This article is distributed under the terms of the Creative Commons Attribution 4.0 International License (http://creativecommons.org/licenses/by/4.0/), which permits unrestricted use, distribution, and reproduction in any medium, provided you give appropriate credit to the original author(s) and the source, provide a link to the Creative Commons license, and indicate if changes were made. The Creative Commons Public Domain Dedication waiver (http://creativecommons.org/publicdomain/zero/1.0/) applies to the data made available in this article, unless otherwise stated. 
genes and proteins in normal appearing mucosa from CRN patients [1-4].

\section{Wnt/ $\beta$-catenin-, ERK/MAPK- and PI3K/Akt pathways in colorectal cancer}

Among the frequently disrupted signaling pathways, perturbation of the Wnt/ $\beta$-catenin pathway has been identified in early lesions of colorectal epithelium. Concurrently, Wnt $/ \beta$-catenin perturbation is regarded a major initiating event in development of CRN as its tumor-suppressor gene adenomatous polyposis coli (APC) is inactivated in about $80 \%$ of sporadic colorectal cancers, CRC. APC inactivation leads to increased nuclear transfer of $\beta$-catenin with formation of a constitutively active $\beta$-catenin-T-cell factor complex [5]. In addition to perturbation of Wnt/ $\beta$ catenin pathway, two other major pathways, the extracellular signal-regulated kinase/mitogen activated protein kinase (ERK/MAPK) and phosphoinositide 3-kinase/Akt (PI3K/Akt)-signaling pathways, are frequently overactive in CRC. Activation of these pathways stimulate cell growth, proliferation and survival [6,7]. As such, constitutive activation of the ERK/MAPK pathway occurs via mutations in KRAS in about $50 \%$ of CRCs [8]. Less frequently, dysregulation of PI3K/Akt pathway is observed following mutations in the CA subtype of the phosphoinositide-3-kinase gene (PIK3CA) or in the PI3K/Akt-related phosphatase and tensin homolog (PTEN) $[9,10]$.

\section{Prostaglandin E2 in colorectal carcinogenesis}

During carcinogenesis the regulatory system of cell cycle is affected by both internal genetic alterations and external signaling. In particular, cytokines, growth factors and eicosanoids trigger signal transduction cascades through receptor tyrosine kinases and $G$ protein-coupled receptors e.g., epidermal growth factor via its receptor EGF and $\mathrm{PGE}_{2}$ through prostanoid receptors type 1-4. Among the prostanoids, prostaglandin $\mathrm{E}_{2}\left(\mathrm{PGE}_{2}\right)$ has been identified as the principal entity promoting cell growth and survival in CRN. It is believed that $\mathrm{PGE}_{2}$ execute these effects via the PI3K/Akt-, ERK/MAPKand $\mathrm{Wnt} / \beta$-catenin pathways [11]. Induction of $\mathrm{PGE}_{2}$ signaling is also well-established as an early and critical step in development of CRN and in tumor progression $[12,13]$. All these pathways and $\mathrm{PGE}_{2}$ metabolism are schematized in Fig. 1.

\section{Hypothesis and aim of study}

We hypothesized that up-regulation of some specific CRC-associated kinases and $\mathrm{PGE}_{2}$-related proteins occur in normal appearing colonic mucosa. The aim of this study was to test this 0 -hypothesis by screening colonic mucosa for a panel of 18 specific genes, all proven altered and involved in CRC development. Tissue samples were endoscopic biopsies obtained from patients with and without CRN. Indeed, identifying perturbation of some of these signaling networks might give further insight into colorectal carcinogenesis per se and also identify potential predictive biomarkers.

\section{Materials and methods Study population}

Patients $\geq 50$ years of age, referred for colonoscopy on suspicion for colorectal disease, were screened for enrollment in the study. Patients with a history of CRN, or CRN detected during colonoscopy, were included in CRN group. Patients with no present or history of CRN represents the control group. Patients were excluded if they received weekly medications in the form of nonsteroidal anti-inflammatory drugs, paracetamol, systemic corticosteroids and/or cytostatics. Furthermore, incomplete colonoscopy, inflammatory bowel disease, malabsorption or previous sigmoid resection and exposure to radiation or chemotherapy within the last year precluded enrollment. Finally, patients were also excluded if diagnosed with any of the genetic CRN syndromes (e.g. adenomatous polyposis coli and hereditary nonpolyposis colorectal cancer), as the focus of this study was sporadic CRN. Risk stratification of adenomas was performed based on number, size and histology grade in accordance with the European guidelines for quality assurance in CRC screening and diagnosis [14].

\section{Biopsy procedure}

Six biopsies from each patient were obtained during endoscopy from endoscopically normal appearing mucosa using standard biopsy forceps (Boston Scientific, Radial Jaw 4, outside diameter of $2.2 \mathrm{~mm}$ ). Biopsies were obtained approximately $30 \mathrm{~cm}$ orally from the anal verge on retraction of the endoscope and at least $10 \mathrm{~cm}$ from endoscopically abnormal appearing mucosa.

\section{Statistical analysis}

Values were expressed as the mean \pm SEM and as fold change. Data was analyzed using two-tailed unpaired t-test. A $p$-value less than 0.05 was considered statistically significant. Bonferroni correction was applied. Calculations were performed using Microsoft Excel 2007 and GraphPad Prism 6.01.

\section{qPCR}

Two biopsies, obtained from each patient, were stored in RNAlater (Thermo Fisher Scientific, Wilmington, DE, USA) at $-80^{\circ} \mathrm{C}$. Biopsies were homogenized using a TissueLyser II (Qiagen, Copenhagen, Denmark), and RNA was extracted using RNeasy Mini Kit (Qiagen, Copenhagen, Denmark). RNA concentration and purity were determined using a NanoDrop 2000 (Thermo Fisher Scientific, 


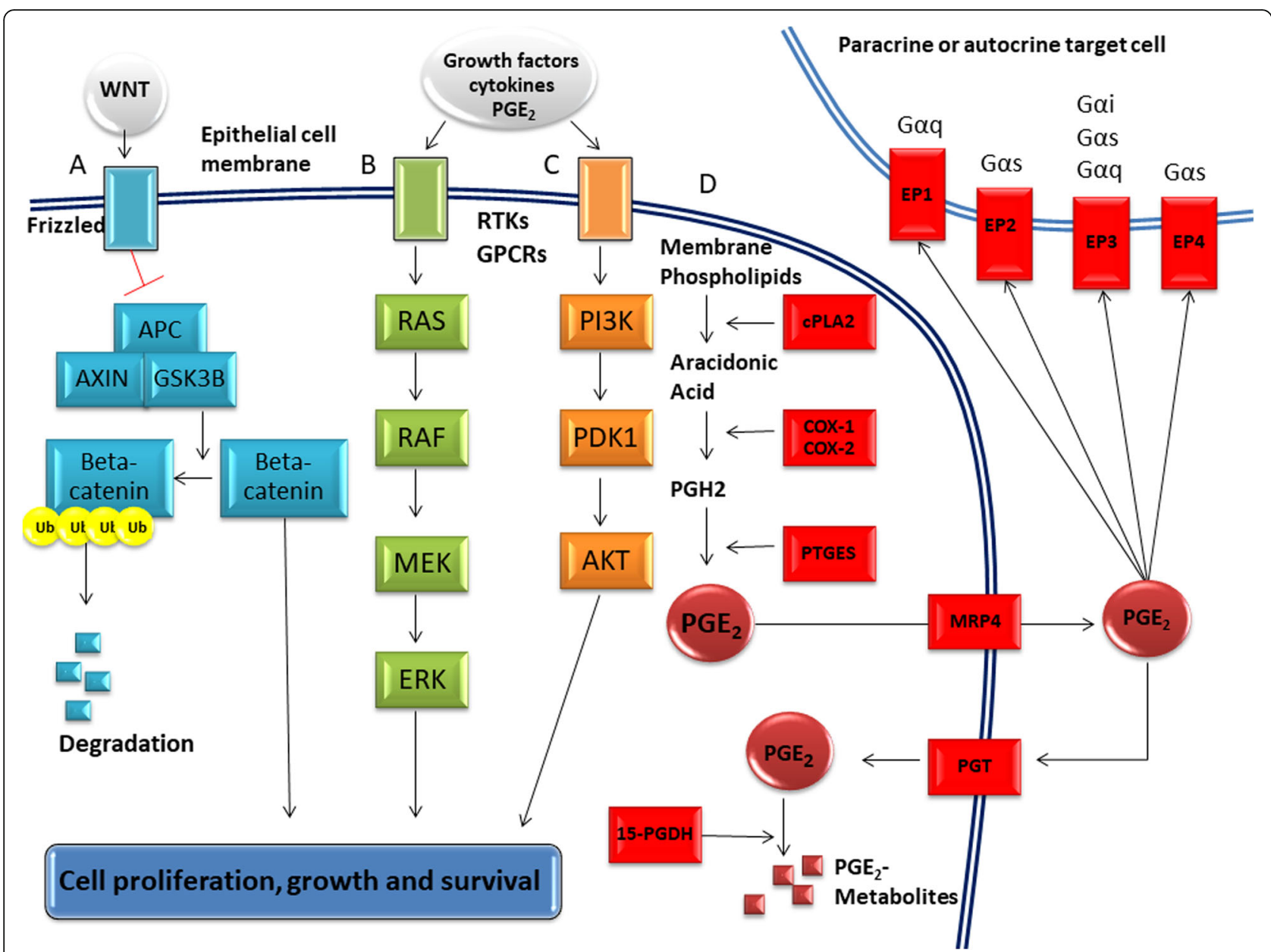

Fig. 1 Simplified schematic illustration of pathways for Wnt/ß-catenin, ERK/MAPK and PI3K/Akt and PGE ${ }_{2}$-metabolism. A) Canonical Wnt/B-catenin signaling. The engagement of the Wnt receptor, Frizzled, leads to the inhibition of the $\beta$-catenin destruction complex, composed of APC, axin and GSK3 $\beta$. $\beta$-catenin thereby avoids ubiquitination and subsequent degradation, thus allowing it to translocate to the nucleus to activate an array of regulatory genes. B) The RAS/RAF/MEK/ERK MAPK pathway. Stimulation of the receptor tyrosine kinase (RTK) or G-protein coupled receptors (GPCRs) leads to sequential activation of RAS, RAF, MEK, and ERK causing modification of substrates promoting cell survival and proliferation. C) In the PI3K/Akt pathway, activation of the RTK or GPCRs leads to sequential modification of phosphatidyl inositol residues of the phospholipid bilayer. In this process, PI3K generates PIP3. PIP3 in association with PDK1 activates Akt. Akt then modulates the activity of downstream substrates including mTOR, thus promoting proliferation and cell survival. D) $\mathrm{PGE}_{2}$-metabolism. $\mathrm{PGE}_{2}$-synthesis begins with catalytic hydrolysis of membrane phospholipids by cytoplasmic phospholipase $\mathrm{A} 2(\mathrm{CPLA})$, thus releasing arachidonic acid (AA). By the action of the COX-1 and COX-2, AA is converted to prostaglandin $\mathrm{H}_{2}\left(\mathrm{PGH}_{2}\right)$. $\mathrm{PGH}_{2}$ is then converted to $\mathrm{PGE}_{2}$ by prostaglandin $\mathrm{E}$ synthase (PTGES). The main exporter of $\mathrm{PGE}_{2}$ is thought to be multi-drug resistance related polypeptide 4 (MRP4). Removal of $\mathrm{PGE}_{2}$ from the extracellular compartment around target cells occurs by diffusion to the blood stream and subsequent uptake and degradation in lung, liver or kidney endothelial cells or by import to colonic epithelial cells through the prostaglandin transporter (PGT) and subsequent degradation by 15-prostaglandin dehydrogenase (15-PGDH). Through autocrine and paracrine signaling, extracellular PGE 2 stimulates the prostaglandin receptors EP1-4. The EPs are GPCRs with EP1 being Gaq-coupled while EP2 and EP4 are Gas-coupled. EP3 is capable of coupling with different G-proteins including Gai, Gas and Gaq

Wilmington, DE, USA), the latter by $\mathrm{A}_{260} / \mathrm{A}_{280}$ and $\mathrm{A}_{260} /$ $\mathrm{A}_{230}$ absorbance ratios. RNA was converted to cDNA using the iScript ${ }^{\text {tw }}$ cDNA Synthesis Kit (BioRad, Copenhagen, Denmark) according to the manufacturers protocol. Primers against genes of interest were designed, synthesized and quality controlled by Primerdesign Ltd. (Primerdesign Ltd., Chandler's Ford, UK). Primers against $\beta$-actin were designed and synthesized by Section of Endocrinology Research, Department of Biomedical Sciences, University of
Copenhagen, primerbank reference 4501885a1. Primer sequences are listed in Table 1. cDNA was amplified on a 7900HT Fast Real-Time PCR System (Applied Biosystems, Foster City, CA, USA) using Fast SYBR ${ }^{\circ}$ Green Master Mix (Applied Biosystems) in accordance with the manufacturers protocol. All samples were run in triplicate with $\beta$-actin primers on all plates. Results were analyzed using SDS 2.4 (Applied Biosystems), and expression was calculated by the $2^{-\Delta \mathrm{Ct}}$ method. 
Table 1 Quantitative real-time PCR primer sequences

\begin{tabular}{|c|c|c|}
\hline Target & Forward $5^{\prime} \rightarrow 3^{\prime}$ & Reverse $5^{\prime} \rightarrow 3^{\prime}$ \\
\hline$\beta$-actin & CATGTACGTTGCTATCCAGGC & CTCCTTAATGTCACGCACGAT \\
\hline PLA2G4A & GAAAAGTGGGCTAAAATGAACAAG & GGGCAATCTTTCTCCATATCAG \\
\hline $\operatorname{COX} 1$ & CGTGTGTGTGACCTGCTGAA & GTACTCCTCGATGACAATCTTGATG \\
\hline $\operatorname{COX} 2$ & CAGGCTTCCATTGACCAGAG & TTTCTCCTGTAAGTTCTTCAAATGAT \\
\hline PTGES & GTGGCTATACCTGGGGACTT & AATCCAAGGGGCTAAGAAACAT \\
\hline EP1 & ATCTGCTGGAGCCCAATGC & GATCTGGTTCCAGGAGGCAA \\
\hline EP2 & CAGACCCTGGTGGCACTG & CGAAGAGCATGAGCATCGTG \\
\hline EP3 & TCAATCAGACATCAGTTGAGCAC & TTTCTTAACAGCAGGTAAACCCAA \\
\hline EP4 & AGTTGGAGCGAGAAGTCAGTA & GCGGCAGAAGAGGCATTTG \\
\hline 15-PGDH & AGTAGTGAACATCAATGAACATCTGA & ACTGGGCAAACCGACATCAT \\
\hline PGT & GCCACAGCAGATGAAGCAAG & CCACCAGGACGAAGAGTGAG \\
\hline ERK1 & AGAGATCATGCTGAACTCCAAG & GTGCTTGCCAGGGAAGATG \\
\hline ERK2 & TGGATGTGGTGTTATGGAAAGA & AAGCAGAGACGCAGAATGAC \\
\hline AKT1 & GGCACCCCTTCCTCACAG & GGCGTACTCCATGACAAAGC \\
\hline AKT2 & CTGCGGAAGGAAGTCATCATT & GGTCGTGGGTCTGGAAGG \\
\hline$\beta$-catenin & ССАТTACAACTCTCCACAACCT & GAGCAAGGCAACCATITCTG \\
\hline GSK3B & AAGTAATCCACCTCTGGCTACC & AGAAGCAGCATTATTGGTCTGTC \\
\hline PPP2R1B & GGTTCACCTCTCGCACATCT & TCTGAGCACAAGGAACGGAAT \\
\hline PTPRM & ACTCGTTGCCACAGTTATAATCTC & GTGTATGGTGACAGGTTAGTGATC \\
\hline
\end{tabular}

Left column: Names of all qPCR-analysed targets. Middle column: Forward primer sequences written $5^{\prime} \rightarrow 3^{\prime}$. Right column: reverse primer sequences written $5^{\prime} \rightarrow 3^{\prime}$.

\section{Western blot and immunohistochemistry Primary antibodies for Western blots and immunohistochemistry}

The following antibodies were employed to detect target antigens by Western blot and immunohistochemistry (IHC), in parenthesis (antibody designator, dilution): goat anti-COX-1 (C-20, 1:800), mouse anti- $\beta$-catenin (E-5, 1:200) and mouse anti-PGDH (H-3, 1:250) were all purchased from Santa Cruz Biotechnology, Heidelberg, Germany. Rabbit anti-ERK1/2 (9102S, 1:1000), rabbit anti-pERK1/2 (9101S, 11,000) and rabbit anti-panAkt (C67E7, 1:1500) were all from Cell Signaling, Leiden, The Netherlands. Rabbit anti-COX-2 (SP21, 1:500) was from Spring Biosciences, Pleasanton, CA, USA. We did not succeed in detecting EP3, neither in Western blots nor in IHC staining, despite using four different antibodies. The following antibodies were tested: Mouse antiEP3 antibody (5F5), sc-57,105 (Santa Cruz Biotechnology), Rabbit anti-EP3 antibody, 101,760 (Cayman Chemicals, Ann Arbor, MI, USA), Rabbit anti-EP3 antibody, APR-065 (Alomone Labs, Jerusalem, Israel), Rabbit anti-EP3 antibody (PTGER3), NBP1-84835 (Bio-techne, Abingdon, United Kingdom). In addition, attempts to detect cytoplasmic phospholipase A2 alpha (cPLA2A) using two different antibodies (Santa Cruz Biotechnology, sc-454 and Cell Signaling 2832) and protein phosphatase 2 isoform beta of scaffold subunit A (PPP2R1B) using mouse anti-PPP2R1B (Santa Cruz Biotechnology, sc-13, 600) proved unsuccessful in both Western blots and IHC. IHC staining for PGT and for COX-2 with two different antibodies (Cayman Chemicals, 160,126 and Spring Biosciences SP21) was attempted, but was unsuccessful.

\section{Homogenization and solubilization of colonic biopsies for Western blot analysis}

Snap-frozen biopsies were homogenized for $20 \mathrm{~s}$ at 5000 $\mathrm{rpm}$ in $150 \mu \mathrm{l}$ buffer $(50 \mathrm{mM}$ TrisHCl, pH 8.5, $5 \mathrm{mM}$ EDTA, $150 \mathrm{mM} \mathrm{NaCl}, 10 \mathrm{mM} \mathrm{KCl}, 1 \%$ Triton X-100, $5 \mathrm{mM} \mathrm{NaF}, 5 \mathrm{mM} \beta$-glycerophosphate, $1 \mathrm{mM}$ sodiumorthovanadate and Complete Protease Inhibitor Cocktail Tablet (Roche, Hvidovre, Denmark)) using a PreCellys 24 (Bertin Instruments, Montigny-le-Bretonneux, France) and ceramic beads (mix of $1.4 \mathrm{~mm}$ and $2.8 \mathrm{~mm}$ beads, VWR, Soeborg, Denmark). Solubilization was continued for $2 \mathrm{~h}$ in the same buffer at $4{ }^{\circ} \mathrm{C}$ with rotation. The solubilized samples were spun for $15 \mathrm{~min}$ at $15000 \mathrm{~g}$ and the resulting supernatant collected for further protein determination and Western blot analysis.

\section{Western blotting}

Twenty-four biopsies were analyzed by Western blotting (12 CRN, 12 controls). $12.5 \mu \mathrm{g}$ protein was separated on 
4-20\% gradient mini-PROTEAN ${ }^{\ominus}$ TGX $^{\mathrm{Tm}}$ Gels (Bio-Rad Laboratories, Copenhagen, Denmark) using the Bio-Rad Laboratories minigel system. Proteins were transferred onto an Immobilon-FL Transfer Membrane $45 \mu \mathrm{M}$ (Merck Millipore, Hellerup, Denmark) in $25 \mathrm{mM}$ Tris base, $200 \mathrm{mM}$ glycine, 20\% methanol, 1\% SDS using the same system. After transfer, membranes were stained with REVERT ${ }^{\mathrm{\tau}}$ Total Protein Stain (LI-COR Biosciences, Cambridge, United Kingdom) and the staining captured using the Odyssey CLx Imaging System (LI-COR Biosciences) to adjust for protein loading (internal loading control). Membranes were subsequently blocked for $1 \mathrm{~h}$ at room temperature in blocking buffer (Odyssey Blocking Buffer (PBS), LI-COR Biosciences). Primary antibody was applied overnight at $4{ }^{\circ} \mathrm{C}$ in blocking buffer. IRDye ${ }^{\circ} 800 \mathrm{CW}$ Secondary Antibodies (1:10000 dilution in blocking buffer, LI-COR Biosciences) were then applied for $30 \mathrm{~min}$ at room temperature and bound antibody detected with the Odyssey CLx Imaging System (LI-COR Biosciences). Western blot was repeated twice on the same samples to ensure reproducibility. Signals originating from target proteins were quantified in Image Studio Version 3.1 using REVERT $^{\mathrm{TM}}$ Total Protein Stain as internal loading control. The target protein signal in each lane was normalized to the total protein stain in the corresponding lane. The normalized mean target protein signal originating from the control group was then set to 1 and all values expressed relative to this value.

\section{Immunohistochemistry}

Biopsies were fixed immediately in $4 \%$ formaldehyde and kept in the formaldehyde solution at $4{ }^{\circ} \mathrm{C}$ until cutting. Cryoprotection was performed by two successive overnight incubations at $4{ }^{\circ} \mathrm{C}$ in PBS containing first 20\% sucrose and subsequently $30 \%$ sucrose. After cryoprotection, $10-15 \mu \mathrm{m}$ cryostat sections were cut. For staining, sections were subjected to antigen retrieval by boiling in $10 \mathrm{mM}$ citric acid pH 6.0 for 5-10 min. After washing in PBS, unspecific binding was blocked for $30 \mathrm{~min}$ in PBS containing $0.1 \%$ triton X-100 and $0.2 \%$ fish skin gelatine. Primary antibodies were diluted in the same buffer and incubated overnight at $4{ }^{\circ} \mathrm{C}$. Bound primary antibody was detected by incubation in AlexaFluor ${ }^{\odot}$-conjugated secondary antibodies (Thermo Fisher Scientific, Roskilde, Denmark) for $1 \mathrm{~h}$ at room temperature. Nuclei were detected using 4',6-diamidino2-phenylindole (DAPI, Thermo Fisher). Sections were mounted in Prolong Diamond (Thermo Fisher Scientific). IHC staining for cytoplasmic phospholipase A2 alpha (cPLA2A), cyclooxygenase 1 and 2 (COX-1, COX-2), prostaglandin transporter (PGT), 15-hydroxyprostaglandin dehydrogenase (15-PGDH), EP3, $\beta$-catenin, ERK1/2, AKT and protein phosphatase 2 isoform beta of scaffold subunit A (PPP2R1B) was attempted. Only COX-1, 15-PGDH and $\beta$-catenin were reliably detected.

\section{Confocal microscopy}

Confocal images were captured using a Zeiss LSM 710 confocal microscope. Images were captured using either a $\times 20$ objective (NA 0.8 ) or a $\times 63$ oil immersion objective (NA 1.4). Pinhole size was set to 1 , the pixel format was $1024 \times 1024$ and line averaging was employed to reduce noise.

\section{Determination of mucosal contents of $\mathrm{PGE}_{2}$ and prostaglandin E metabolites}

Immediately upon extraction, biopsies were placed in 1 $\mathrm{ml}$ of Tris-buffered Ringer's solution ( $\mathrm{pH}$ 7.4) and snap frozen in liquid nitrogen. The Ringer's solution was composed of (in mM) 20 Tris, $130 \mathrm{NaCl}, 1 \mathrm{CaCl}_{2}, 2.5$ $\mathrm{K}_{2} \mathrm{HPO}_{2}$ and $1 \mathrm{MgSO}_{4}$ as well as protease inhibitor cocktail powder (one bottle dissolved in $100 \mathrm{ml}$ Ringer's solution), from Sigma-Aldrich (Schnelldorf, Germany, cat. no. P2714). In order to inhibit both synthesis and catabolism of $\mathrm{PGE}_{2}$, the Ringer's solution additionally contained $13 \mu \mathrm{M}$ of indomethacin from Sigma-Aldrich (Seelze, Germany) and $110 \mu \mathrm{M}$ of the 15-PGDH-inhibitor 5-[[4-(ethoxycarbonyl)phenyl]azo]-2-hydroxy-benzeneacetic acid (Santa Cruz Biotechnology, Heidelberg, Germany, cat. no. 78028-01-0). $110 \mu \mathrm{M}$ 15-PGDH inhibitor concentration was obtained by diluting a solution of $1 \mathrm{mg}$ inhibitor dissolved in $33 \mu \mathrm{l}$ DMSO to a ratio of 1 DMSO:833 Ringer. Samples were stored at $-80^{\circ} \mathrm{C}$. After a quick thaw, biopsies were retrieved and dabbed twice on Watman filter no 1 and weighed (range 5-15 mg) and suspended in $1 \mathrm{ml}$ Ringer. Samples were then systematically pestel-squeezed to promote breakage of cell membranes and centrifuged for $30 \mathrm{~s}$ at 10,000 rpm in a Beckman microfuge to form a supernatant. Volumes of $50 \mu \mathrm{L}$ supernatant were used for measuring $\mathrm{PGE}_{2}$ as well as the stable $\mathrm{PGE}_{2}$ metabolite PGE-M according to manufacturer's instructions using ELISA kits from Cayman Chemical, Ann Arbor, MI, USA, cat. No. 514010 and 514,531. The ELISA results were adjusted for the weight of sample biopsy. Test for possible interference from the organic solvent DMSO was performed with up to double the maximal Ringeradded concentration of DMSO and demonstrated no shifts in the standard curves. Tests for general interference due to different dilutions of samples were performed and proved no need for adjustments.

\section{Results}

\section{Patient characteristics}

Twenty-seven patients were included. Fourteen patients in CRN group and 13 patients in control group. One additional patient was excluded from analysis due to histologically verified hyperplastic polyps, which are not classified as neoplasia. Median age was 64 years in CRN group and 62 years in control group. CRN group contained $7(7 / 14)$ women versus $8(8 / 13)$ in control group. 
Nine patients $(9 / 14)$ had present $C R N$, while the $5(5 / 14)$ remaining patients had previously been diagnosed with CRN. Three patients (3/14) had primary CRC. The remaining $11(11 / 14)$ patients had tubular adenomas which were risk stratified as being high risk adenomas $(n=3 / 14)$, intermediate risk adenomas $(n=3 / 14)$ and low risk adenomas $(n=5 / 14)$ based on number, size and histology grade in accordance with the European guidelines for quality assurance in CRC screening and diagnosis [14]. Eight patients (8/14) from CRN group and $6(6 / 13)$ patients from control group had one or more comorbidities such as anemia, diabetes, hypothyroidism, cardiovascular disease, asthma, osteoporosis, psoriasis, chronic dermatitis, allergic rhinitis and/or a psychiatric diagnosis. Two patients in CRN group had a history of primary prostate cancer without metastasis. In the control group 4 patients had a history of primary non-colorectal cancer without metastasis: Melanoma ( $n=1 / 14)$, testis seminoma $(n=1 / 14)$, uterus cancer $(n=1 / 14)$ and breast cancer $(n=1 / 14)$. Nine patients $(9 / 14)$ in CRN group and $10(10 / 13)$ patients in control group received medications e.g. anti-thrombotics, angiotensin-converting-enzyme inhibitors, angiotensin-IIreceptor antagonists, $\beta$-blockers, calcium-blockers, statins, diuretics, $\beta 2$-agonists, anti-histamines, insulin, levothyroxine, proton pump inhibitors, laxantia, loperamid, antidepressants, estrogen or bisphosphonates. There were no apparent differences between the CRN and control groups in use of medications nor in co-morbidity profiles. Level changes for ERK, Akt, $\beta$-catenin and PPP2R1B expression.

We screened colonic mucosa for a panel of 18 specific genes, all proven altered and involved in CRC development. All 18 targeted mRNAs were detected. Perturbed expression profile was observed in CRN patients with significantly higher mRNA-expressions measured for the following gene transcripts: PLA2G4A, EP3, ERK1, ERK2, $A k t 1, A k t 2$ and PPP2R1B, Table 2 and Fig. 2.

For the CRN group we detected upregulation of ERK1 $(p=0.007)$ and ERK2 $(p=0.02)$ which are ubiquitous regulators of cellular proliferation, differentiation, survival and transformation. ERK1 and ERK2 were expressed 1.87 and 2.0 times higher, respectively, in CRN patients. ERK1 expression was higher compared to ERK2 in both patient groups. $A k t 1$ and $A k t 2$ were both significantly up-regulated in CRN group ( $p=0.02$ and $p=0.041$, respectively). $\beta$-catenin was highly expressed in both groups and with substantial variability in CRN group. The phosphatase subunit PPP2R1B, which is considered a negative regulator of both ERK and Akt activation and a stimulator of Wnt/ $\beta$-catenin signaling, was significantly up-regulated in CRN group $(p=0.03)$.

\section{Level changes for PLA2G4A and EP3}

Analysis of entities in $\mathrm{PGE}_{2}$ metabolism by qPCR demonstrated expression of PLA2G4A, which encodes a major enzyme involved in arachidonic acid mobilization, was significantly increased in CRN group $(p=0.02)$. With respect to the EP-receptors, EP4 had by far the highest expression, followed by EP2 and EP3, while EP1 showed the lowest expression. Only EP3 was significantly up-regulated in CRN group compared to controls $(p=0.016)$. Of all investigated mRNAs, 15-PGDH (the major enzyme involved in $\mathrm{PGE}_{2}$ degradation), showed the highest expression in both groups and 1.9-fold higher expression in CRN group compared to controls $(p=0.066)$. Expression of $C O X-1$ was higher than $C O X-2$ in both groups (CRN 2.4-fold; controls 2.5-fold), while none of the two COX enzymes were significantly altered between patient groups. When Bonferroni correction was applied none of the 18 investigated genes reached the required significance level of $p<0.0028$.

\section{Trend towards increase in $\beta$-catenin, COX-1, COX-2 and ERK1 protein expression}

To determine whether the changed mRNA expression profile observed in CRN patients was associated with changes in the protein expression level of these targets, we next performed Western blot analysis on colonic biopsies from CRN patients and controls. We successfully detected COX-1, COX-2, 15-PGDH, ERK1/2 and the active phosphorylated ERK1/2 (pERK1/2) proteins as well as AKT (using an antibody that detects AKT1-3) and $\beta$-catenin. We analyzed the protein expression level for each of these targets in the control group (12 biopsies) and compared them to the expression levels in the CRN group (12 biopsies). Relative protein expressions for the detected target proteins are shown in Fig. 3. The Western blot results suggest a trend towards a moderate increase in $\beta$-catenin as well as minor increases in COX-1, COX-2 and ERK1. The observed changes were not, however, statistically significant.

\section{Localization of COX-1, 15-PGDH and $\beta$-catenin}

We next sought to investigate the localization of target proteins by immunohistochemistry. We attempted to detect cPLA2A, COX-1, COX-2, EP3, PGT, 15-PGDH, ERK1/2, AKT, $\beta$-catenin and PPP2R1B in colonic biopsies from CRN patients and controls. The targets were selected to map localizations of proteins involved in different aspects of the $\mathrm{PGE}_{2}$ pathway and to study any differences in protein localizations and/or possible expressions in CRN patients compared to controls. Possibly due to inadequate (human) specificity of the tested antibodies or paucity of target proteins, we failed to reliably detect cPLA2A, COX-2, PGT, EP3, ERK1/2, AKT and PPP2R1B in IHC. Only COX-1, 15-PGDH and $\beta$-catenin were detected. The results are shown in Fig. 4. 15-PGDH displayed strong cytoplasmic staining in epithelial cells at the crypt apex. COX-1 was selectively expressed in a small subset of cells in the epithelial cell 
Table 2 Difference in mRNA expression in CRN versus controls

\begin{tabular}{|c|c|c|c|c|}
\hline & Control mean $( \pm$ SEM) & CRN mean ( \pm SEM) & Fold change & $P$ \\
\hline PLA2G4A & $0.0063( \pm 0.0008)$ & $0.0110( \pm 0.0017)$ & 1.75 & $0.020^{*}$ \\
\hline $\operatorname{COX}-1$ & $0.0054( \pm 0.0007)$ & $0.0069( \pm 0.0009)$ & 1.26 & 0.252 \\
\hline $\operatorname{COX}-2$ & $0.0022( \pm 0.0006)$ & $0.0029( \pm 0.0007)$ & 1.31 & 0.490 \\
\hline PTGES & $0.0008( \pm 0.0001)$ & $0.0010( \pm 0.0002)$ & 1.26 & 0.388 \\
\hline EP1 & $0.000029( \pm 0.0000079)$ & $0.000034( \pm 0.0000064)$ & 1.18 & 0.626 \\
\hline$E P 2$ & $0.0013( \pm 0.0002)$ & $0.0018( \pm 0.0003)$ & 1.33 & 0.207 \\
\hline EP3 & $0.0011( \pm 0.0002)$ & $0.0026( \pm 0.0005)$ & 2.34 & $0.016^{*}$ \\
\hline EP4 & $0.0217( \pm 0.0021)$ & $0.0305( \pm 0.0047)$ & 1.41 & 0.096 \\
\hline$P G T$ & $0.0301( \pm 0.0032)$ & $0.0364( \pm 0.0038)$ & 1.21 & 0.217 \\
\hline 15-PGDH & $0.2283( \pm 0.0572)$ & $0.4350( \pm 0.0921)$ & 1.91 & 0.066 \\
\hline ERK1 & $0.0640( \pm 0.0076)$ & $0.1198( \pm 0.0171)$ & 1.87 & $0.007^{* *}$ \\
\hline ERK2 & $0.0018( \pm 0.0002)$ & $0.0036( \pm 0.0007)$ & 2.00 & $0.020^{*}$ \\
\hline Akt1 & $0.0311( \pm 0.0033)$ & $0.0564( \pm 0.0092)$ & 1.82 & $0.020^{*}$ \\
\hline Akt2 & $0.0193( \pm 0.0020)$ & $0.0283( \pm 0.0035)$ & 1.46 & $0.041^{*}$ \\
\hline$\beta$-Catenin & $0.1694( \pm 0.0197)$ & $0.2662( \pm 0.0486)$ & 1.57 & 0.082 \\
\hline GSK3 $\beta$ & $0.0289( \pm 0.0031)$ & $0.0759( \pm 0.0220)$ & 2.63 & 0.054 \\
\hline PPP2R1B & $0.0196( \pm 0.0022)$ & $0.0317( \pm 0.0048)$ & 1.62 & $0.030^{*}$ \\
\hline PTPRM & $0.0079( \pm 0.0011)$ & $0.0142( \pm 0.0029)$ & 1.81 & 0.059 \\
\hline
\end{tabular}

Control group: $N=13, \mathrm{CRN}$ group: $N=14$. Data are expressed as the mean ( $\pm \mathrm{SEM}$ ) and as fold change of mRNA expression in CRN group compared to control group. ${ }^{*}=p<0.05,{ }^{* *}=p<0.01$

layer where the enzyme displayed a reticular, intracellular localization associated with the perinuclear area. We believe that these COX-1 expressing cells represent epithelial tuft cells $[15,16]$. $\beta$-catenin showed expression throughout the epithelial layer and was mainly associated with lateral membranes of the epithelial cells. No apparent differences in protein localization were observed between CRN and control groups.

\section{Indication of increased prostaglandin E2 content in mucosa of CRN patients}

Mean mucosal $\mathrm{PGE}_{2}$ content $(\mathrm{pg} / \mathrm{mg}$ tissue) was $1234( \pm 98)$ in CRN group and $980( \pm 109)$ in control group, $p=0.095$, Fig. 5. Translated to tissue concentrations, the values come to $3.50 \mu \mathrm{M}$ in CRN and $2.78 \mu \mathrm{M}$ in control group. Mean mucosal $\mathrm{PGE}_{2}$ metabolite content ( $\mathrm{pg} / \mathrm{mg}$ tissue) was 12.58 $( \pm 2.54)$ in CRN group versus $10.90( \pm 2.05)$ in control group.

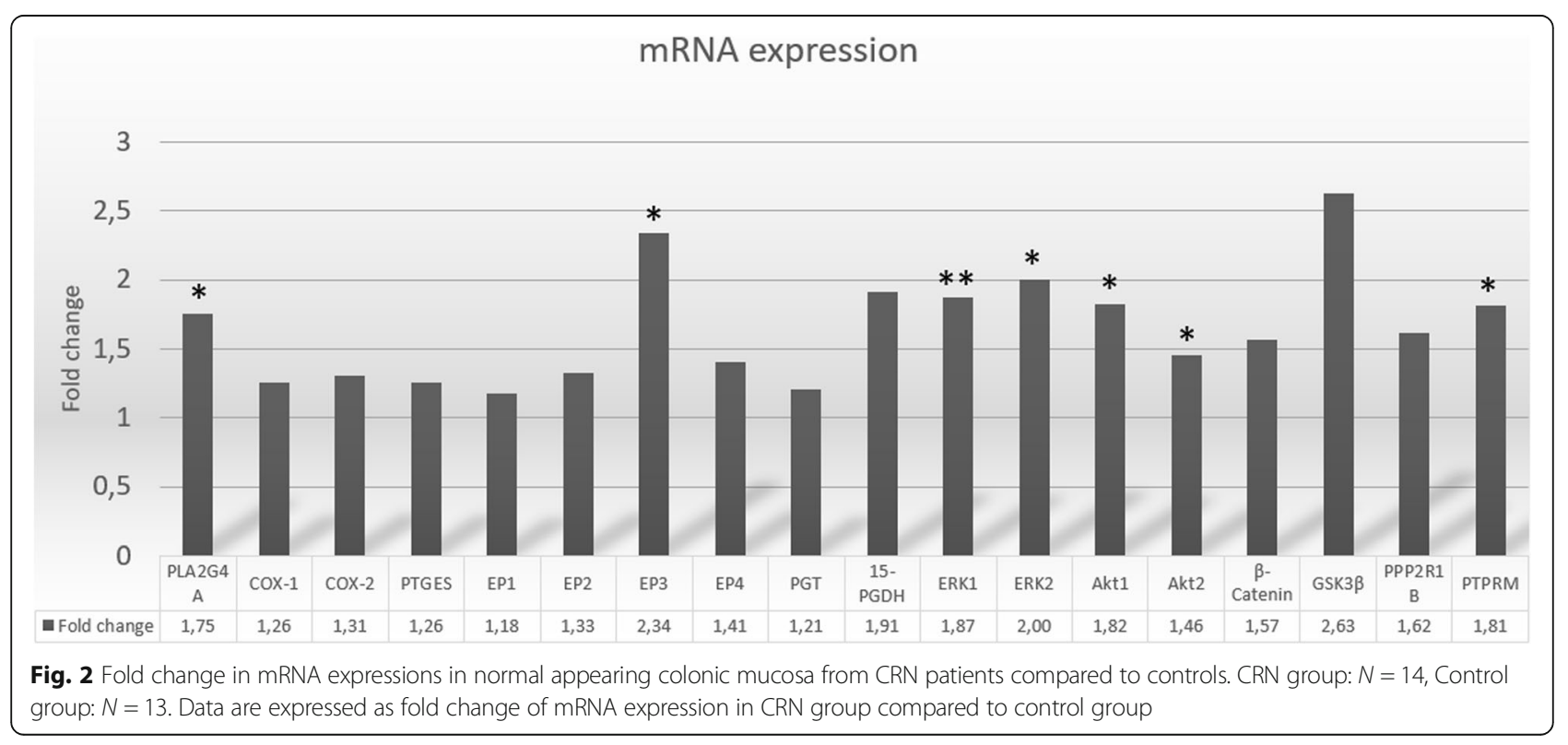




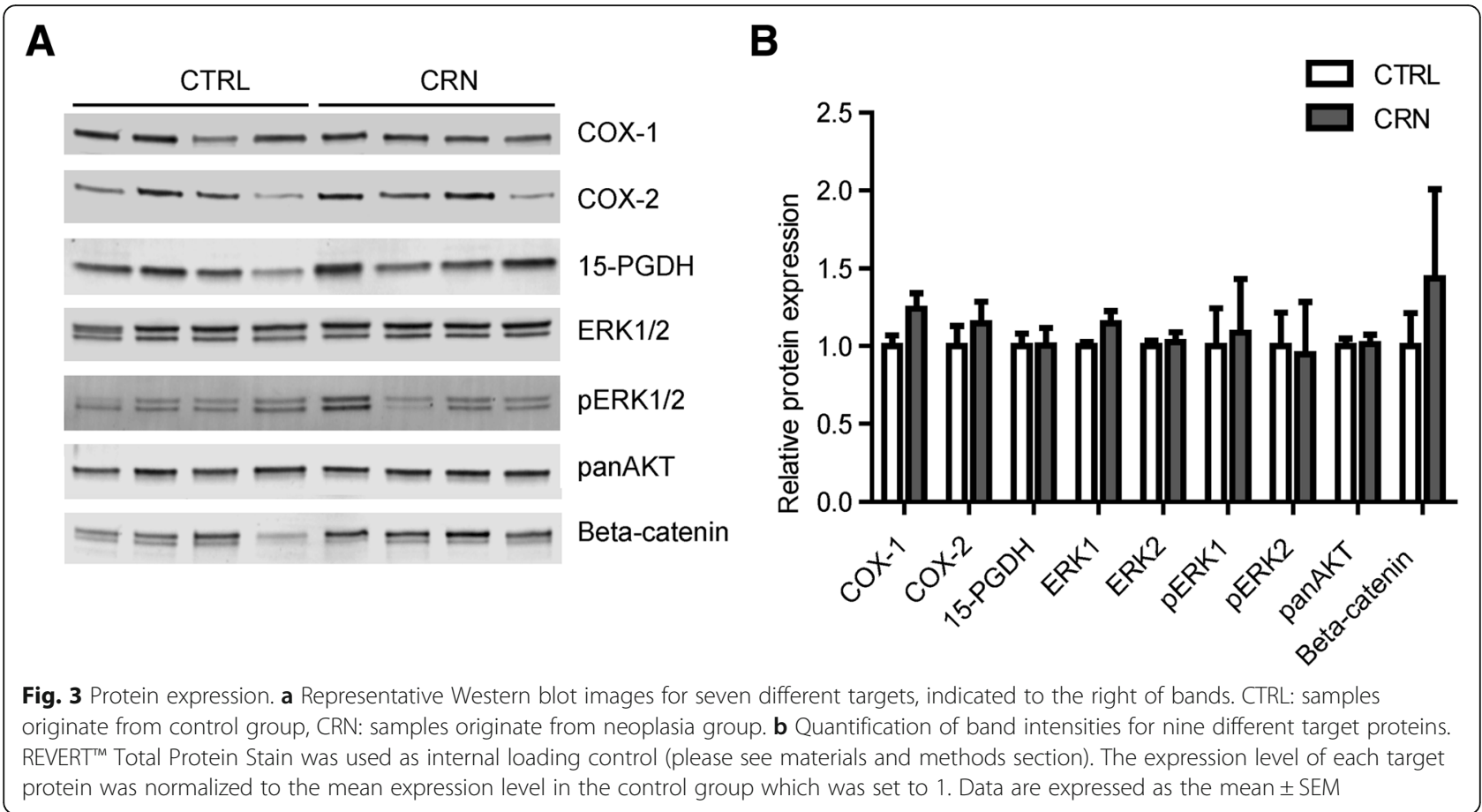

\section{Discussion}

Normal appearing, tumor-remote colonic mucosa in CRN patients has so far been scarcely explored. Here we document significant up-regulation of mRNA expression for the ERK1, ERK2, Akt1, Akt2, PLA2G4A, the prostanoid receptor $E P 3$ as well as for $P P P 2 R 1 B$ in normal appearing colonic mucosa from patients with CRN, Table 2. The range in fold change of these gene expressions was 1.46-2.34. The fact that our observed changes in gene expression were somewhat modest compared to changes reported in cancer tissues was to be expected since we examined normal appearing colon mucosa distant from neoplastic tissue. Furthermore, CRN group encompassed a wide range of stages of CRN and both patients with current CRN as wells as patients with a history of CRN. Since we perceive CRN development as a spectrum from low-grade adenomas to CRC, all CRN data were pooled into one group, tacitly acknowledging that different stages of CRN are likely to contribute differentially to the observed alterations of gene expression. Thus, it was a heterogenous CRN group with a limited number of observations and hence the observed changes between groups were not strong enough to reach the required significance level of a Bonferroni correction. However, our results point towards perturbed gene expression in the normal colon mucosa of CRN patients. These alterations in mRNA expression did not translate into significant alterations at protein level, Fig. 3. Although, the mRNA expressions of the above stated gene transcripts have previously been shown perturbed in CRC affected mucosa, we do not know the precise biological impact of the altered gene expressions in the normal appearing mucosa.

\section{ERK and Akt signaling pathways}

We observed significant up-regulation of mRNA expression for ERK1, ERK2, Akt1 and Akt2 in normal appearing mucosa of CRN patients. Contrary, examining protein abundance, we found a marginal increase of ERK1 $(p=0.09)$ in CRN-group, while phospho-ERK1, ERK2 and panAkt were similar between groups. One might argue that protein expression, especially for phosphorylated proteins, is a more direct marker for cell function compared to mRNA. However, Western blot measurements of protein expression are far less accurate than the qPCR method for measuring mRNA. The observed up-regulation of both ERK and Akt mRNAs are quite interesting findings given the fact that ERK/MAPK and PI3K/Akt pathways are well established important pathways in regulating proliferation and human carcinoma survival [17, 18]. ERK1 and ERK2 are ubiquitous regulators of multiple cellular processes and dysregulated nuclear accumulation of activated ERKs (pERK) can lead to genomic instability and subsequent CRN progression [19-23]. It is difficult to assign a precise role of ERK signaling in human carcinogenesis due to its complexity and dependence on signaling intensity. Still, ERKs may play oncogenic and/or tumor suppressing 


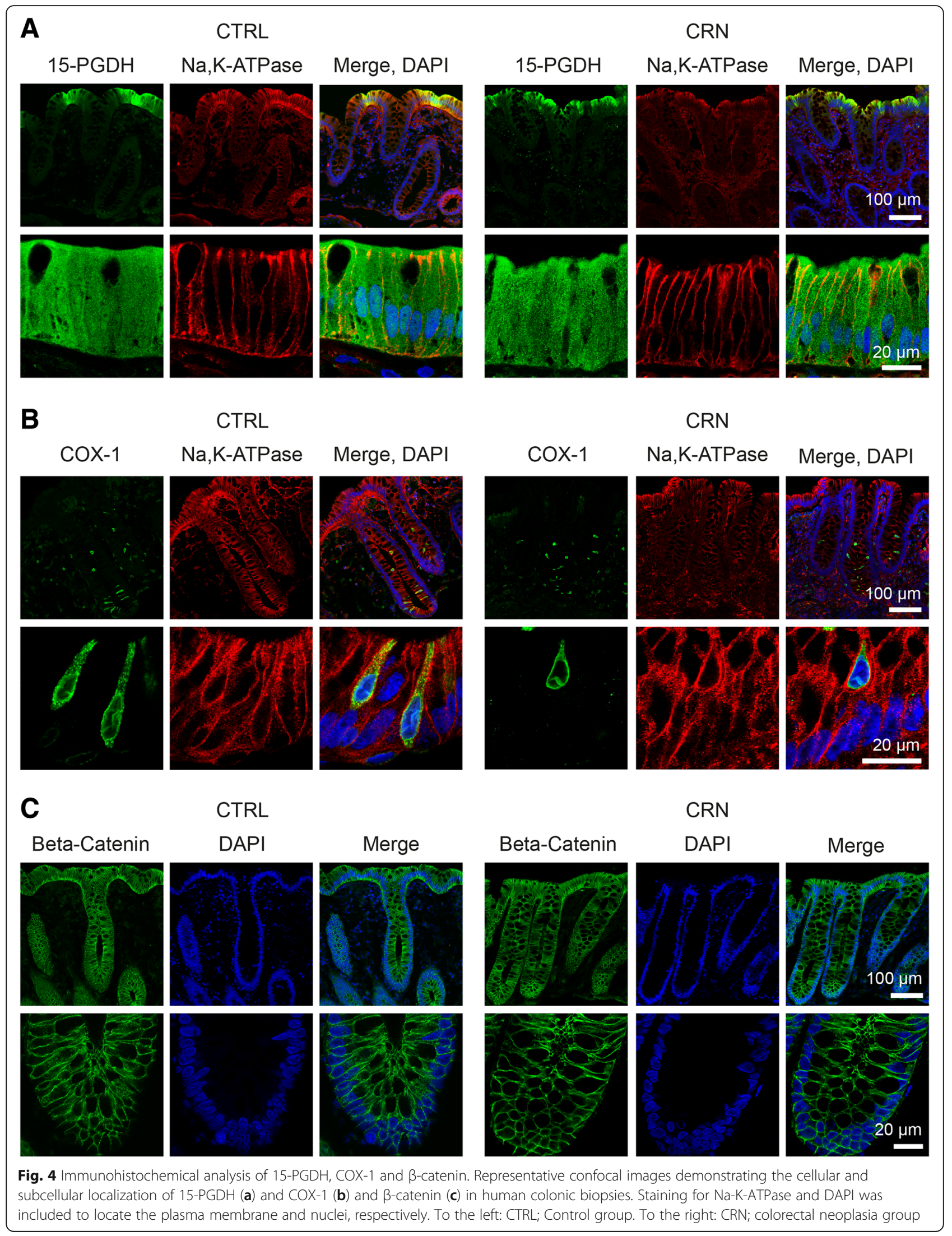




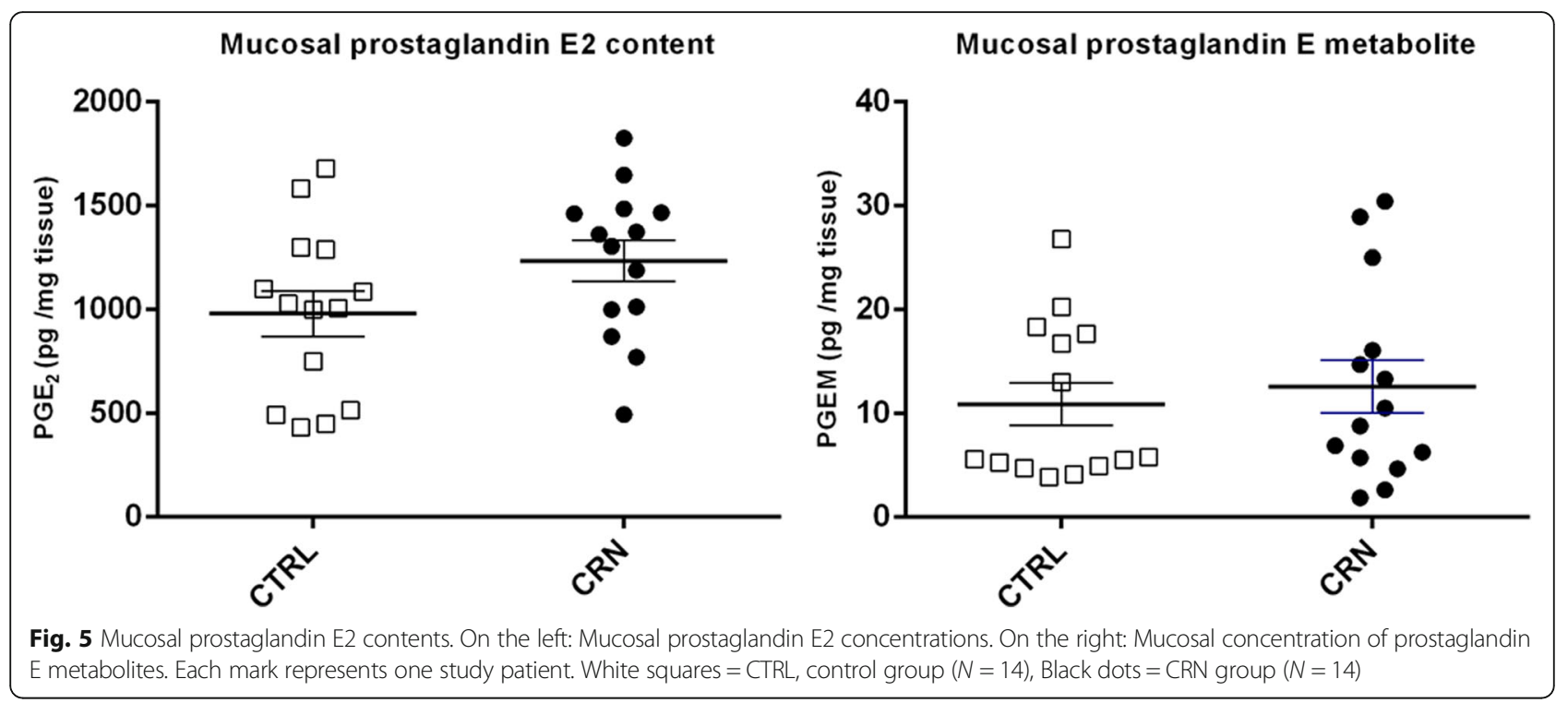

roles in normal appearing colonic mucosa [24-26]. Our findings of markedly lifted expression levels for ERK1 points to this kinase as a possible useful predictive biomarker for CRN development. In addition to ERK and $A k t$, we found enhanced mRNA expression of the structural subunit $A \beta, P P P 2 R 1 B$, which is an isoform of the scaffold subunit of protein phosphatase 2A (PP2A). PP2A is considered a tumor suppressor and a negative regulator of both ERK and Akt activation and stimulator of $\mathrm{Wnt} / \beta$-catenin signaling $[27,28]$. In the present study, PPP2R1B mRNA was up-regulated in CRN group and we speculate that this is a compensatory up-regulation to counteract up-regulation of Akt and ERK.

\section{Prostaglandin E2 signaling}

\section{Prostaglandin E2 synthesis and mucosal content}

As mentioned PLA2G4A mRNA expression is upregulated in normal appearing colonic mucosa from CRN patients, Table 2. The PLA2G4A gene encodes the enzyme cPLA2A which is a key enzyme involved in arachidonic acid mobilization and upstream release of many lipid mediators including lysophospholipids, prostaglandins, leukotrienes and lipoxins [29, 30]. Understanding how the cPLA2A enzyme regulates tumorigenesis is hampered by the entwined effects of its many interacting products of downstream eicosanoid mediators. Meanwhile, our result of up-regulated PLA2G4A could indicate an increased substrate concentration for eicosanoid generation. Thus, despite we did not observe upregulation of COX-1 and -2 enzyme mRNA, a higher substrate level may yield a higher $\mathrm{PGE}_{2}$-concentration. This corroborates with previous reports demonstrating higher sensitivity to indomethacin in CRN patients versus controls $[3,16,31]$. In terms of the resulting mucosal content of active $\mathrm{PGE}_{2}$, our assay indicates that the mean concentration is lifted (26\%) in CRN patients. The observed mucosal $\mathrm{PGE}_{2}$ content in this study may be transformed into tissue concentrations of 3.50 and $2.78 \mu \mathrm{M}$ for CRN and control patients, respectively. Based on these findings and our finding of a highaffinity EP4 subtype receptor (estimated $\mathrm{EC}_{50}$ of $10 \mathrm{nM}$ ) in similar patient mucosal tissue samples (S. Kjaergaard et al., unpublished; U.R. Feddersen et al., unpublished), we assume that the majority of $\mathrm{PGE}_{2}$ is to be found intracellularly and that the extracellular mucosal concentrations are 3 orders of magnitude lower. Taken together, findings suggest presence of a mild chronic inflammation in endoscopically normal appearing mucosa of CRN patients. A similar $27 \%$ marginal increase $(p=0.09)$ in mean colonic mucosal $\mathrm{PGE}_{2}$ concentration was reported by Krishnan et al. for patients treated for CRN [32].

\section{Prostaglandin E2 receptors}

Investigating EP receptors, we found EP3 to be upregulated in CRN patients, Table 2. Interestingly, a study of paired colonic normal and tumor tissues from CRN patients, reported the tumor tissue had downregulated expression of EP3 mRNA [33]. Of note, the EP3 receptor is unique among EP receptor subtypes, in that there are multiple isoforms generated through alternative mRNA splicing in the carboxyl tail of the EP3 gene resulting in isoform specific differences in G-protein coupling and signaling [34]. Our EP3 qPCR-primer did not differentiate between EP3 isoforms. Nonetheless, the major EP3 isoform is thought to couple to an inhibitory $\mathrm{G}$ protein (Gi), and hence the major outcome of $\mathrm{PGE}_{2}-\mathrm{EP} 3$ 
receptor signaling is inhibition of adenylate cyclase and activation of the ERK/MAPK pathway [35]. Thus, the role of the EP3 receptor in tumorigenesis seems to be multifaceted and isoform-dependent. As for cPLA2A, azoxymethane-induced colon cancer development is enhanced in EP3 receptor knockout mice, suggesting an antitumorigenic function for the EP3 receptor [33, 36]. Further studies of the mRNA and protein expressions of individual EP3 isoforms in normal appearing colonic mucosa from humans are warranted.

\section{Prostaglandin E2 influx and degradation}

In terms of cellular $\mathrm{PGE}_{2}$ influx and subsequent elimination we found no change in mRNA expressions of the major and specific $\mathrm{PGE}_{2}$ influx transporter $P G T$ in CRN patients. The expression of $P G T$, an organic anion polypeptide transporter (OATP) has been reported downregulated in CRC tissue [37]. However, other specific $\mathrm{PGE}_{2}$ OATP-transporters, potentially involved in regulatory removal of $\mathrm{PGE}_{2}$, were not investigated in the present study. However, our group reported compensatory increase in the level of two OATP $\mathrm{PGE}_{2}$ influx transporters, OATP2B1 and OATP4A1, located in the basolateral membrane of human colonic epithelia from CRN patients [38]. Since lowered expression of the $\mathrm{PGE}_{2}$ inactivating enzyme, 15-PGDH, has been observed in cancer cells [39], we hypothesized a decrease in 15-PGDH expression in CRN patients compared to controls. Meanwhile, this hypothesis was not supported by a nearly 2-fold $(p=0.066)$ increase in 15-PGDH mRNA expression in CRN patients. The mechanism(s) for the observed elevation of 15-PGDH mRNA expression in the normal appearing colonic mucosa of CRN patients is unresolved. We speculate that a negative feedback balance between increased $\mathrm{PGE}_{2}$ production and subsequent increase in removal/inactivation is present in the normal appearing mucosa of CRN patients.

\section{Is our finding of perturbed mRNA expression a CRN predisposition?}

Our suggestions of perturbed mRNA expressions as possible predisposing factors for the individual development of CRN could also be explained by the extant tumor tissue per se. Thus, at play could be local paracrine inducers or more systemic neuro-, endo- or immunocrine signaling from tumor to neighboring colonic mucosal areas. Yet, another pathway might be special cancer-inducing environmental stimulants as products of food digestion or microbial activity affecting gene expression in non-tumor mucosa. A conclusive settlement of this question between inborn predisposing constitutions versus tumor activity or environmental factors in normal appearing epithelium will require larger population studies. Regardless of the underlying mechanisms, our observation of up-regulation of several genes in normal appearing colonic mucosa suggests that normal appearing mucosa of CRN patients differs from nonCRN patients at a molecular level. Since up-regulated genes in this study have been found perturbed in CRC studies on colonic biopsies of affected mucosa, they could indicate possible predispositions for CRC development. The exploratory nature of our relatively small study cohort points to a need for further confirmation in larger prospective studies in order to determine if the observed aberrant marker genes may be useful predictive biomarkers.

\section{Conclusion}

We observed significant up-regulation of ERK1, ERK2, $A k t 1, A k t 2$, PLA2G4A, prostanoid receptor EP3 and phosphatase scaffold subunit $P P P 2 R 1 B$ mRNA expression in normal appearing colonic mucosa of patients with CRN. Accordingly, normal appearing mucosa of CRN patients differs from non-CRN patients at a molecular level. Most notably, mRNA expression of ERK1 was lifted with high significance of $p=0.007$ and may therefore be considered a potential candidate gene as predictive biomarker for developing CRN. Our observations need to be validated in larger prospective studies.

\section{Abbreviations \\ 15-PGDH: 15-hydroxyprostaglandin dehydrogenase; Akt1 and 2: Subtypes 1 and 2 of the Akt protein kinase (protein kinase B); APC: Adenomatous polyposis coli; COX-1 and COX-2: Cyclooxygenase subtypes 1 and 2; CPLA2A: Cytosplamic phospholipase A2 alpha; CRC: Colorectal cancer; CRN: Colorectal neoplasia; EP1, EP2, EP3 and EP4: PGE receptor subtypes EP 1-4; ERK1 and 2: Subtypes 1 and 2 of the extracellular signal-regulated kin- ase; GPCR: G-protein coupled receptor; GSK3ß: Glycogen synthase kinase 3 beta; MAPK: Mitogen activated protein kinase; mTOR: Mammalian target of rapamycin; OATP: Organic anion polypeptide transporter; $\mathrm{PGE}_{2}$ : Prostaglandin E2; $\mathrm{PGH}_{2}$ : Prostaglandin H2; PGT: Prostaglandin transporter; \\ PI3K: Phosphoinositide 3-kinase; PIK3CA: CA phosphoinositide-3-kinase gene; PIP3: Phosphatidyl inositol triphosphate; PLA2G4A: Phospholipase A2 group IVA; PP2A: Protein phosphatase 2A; PPP2R1B: Isoform beta of scaffold subunit A for protein phosphatase 2; PTEN: Phosphatase and tensin homolog; \\ RTK: Receptor tyrosin kinase}

\section{Acknowledgements}

We wish to acknowledge associate professor Bolette Hartmann for ELISA assistance and PhD student Rasmus Hytting-Andreasen for assistance in preparing RNA-extraction and in obtaining GPCR reference genes. We thank Nancy Thomsen Mutsaers for excellent technical assistance and acknowledge the Core Facility for Integrated Microscopy, Faculty of Health and Medical Sciences, University of Copenhagen. We thank all participating patients for tissue donations as well as the staff at endoscopy unit of Digestive Disease Center at Bispebjerg Hospital for general support.

\section{Authors' contributions}

CHP was the principal investigator and took part in all aspects of this study and was the principal contributor in writing the manuscript. BM contributed to the study design and was a contributor in writing the manuscript. CB was a contributor in performing Western blots and analyzing Western blot data. HBR contributed by performing and analyzing Western blot and IHC data. TD contributed by performing PCR and analyzing PCR data. MBH served as the supervisor of the project and contributed in writing the manuscript. NB 
made a major contribution in designing the study and in writing the manuscript. All authors read and approved of the final manuscript.

\section{Funding}

This work was kindly supported by The Danish Cancer Society (jr. no. R141-A8964-15-S7), Else and Mogens Wedell-Wedellborgs Foundation (jr. no. 30-17-1), and Civilingeniør Bent Bøgh og Hustru Inge Bøghs Fond. The above listed funding supporters have contributed with financial support exclusively and did not have any influence on the design, experimental data, data interpretation or writing of paper.

\section{Availability of data and materials}

The datasets used and/or analyzed during the current study are available from the corresponding author on reasonable request.

\section{Ethics approval and consent to participate}

The study protocol was approved by The Scientific Ethical Committee of Copenhagen (H32013107) and The Danish Data Protection Agency (BBH-2013-024, I-Suite nr: 02342) and conducted in accordance with the Helsinki declaration. All patients participating gave prior informed written consent.

\section{Consent for publication}

Not applicable.

\section{Competing interests}

Mark Berner-Hansen was also an employee at Zealand Pharma, Denmark. All authors have no conflict of interest related to the present study.

\section{Author details}

'Digestive Disease Center K, Bispebjerg Hospital, DK-2400 Copenhagen, Denmark. ${ }^{2}$ Department of Biomedical Sciences, Faculty of Health Sciences, University of Copenhagen, DK-2200 Copenhagen, Denmark.

Received: 19 June 2018 Accepted: 13 June 2019

Published online: 28 June 2019

\section{References}

1. Polley A, Mulholland F, Pin C, Williams EA, Bradburn DM, Mills SJ, Mathers JC, Johnson IT. Proteomic analysis reveals field-wide changes in protein expression in the morphologically Normal mucosa of patients with colorectal neoplasia. Cancer Res. 2006;66:6553-62.

2. Chen LC, Hao CY, Chiu YS, Wong P, Melnick JS, Brotman M, Moretto J, Mendes F, Smith AP, Bennington JL, Moore D, Lee NM. Alteration of gene expression in normal-appearing colon mucosa of APC (min) mice and human cancer patients. Cancer Res. 2004;64:3694-700.

3. Mahmood B, Damm M, Jensen T, et al. Phosphodiesterases in non-neoplastic appearing colonic mucosa from patients with colorectal neoplasia. BMC Cancer. 2016;16:938.

4. Damm MMB, Jensen TSR, Mahmood B, Lundh M, Poulsen SS, Bindslev N, Hansen MB. Acetylcholine-related proteins in non-neoplastic appearing colonic mucosa from patients with colorectal neoplasia. Mol Carcinog. 2017;56:2223-33.

5. Goss KH, Groden J. Biology of the adenomatous polyposis coli tumor suppressor. J Clin Oncol. 2000;18:1967-79.

6. Downward J. Targeting RAS signalling pathways in cancer therapy. Nat Rev Cancer. 2003:3:11-22.

7. Vivanco I, Sawyers CL. The phosphatidylinositol 3-kinase AKT pathway in human cancer. Nat Rev Cancer. 2002:2:489-501.

8. Bos JL, Fearon ER, Hamilton SR, Verlaan-de Vries M, van Boom JH, van der Eb AJ, Vogelstein B. Prevalence of ras gene mutations in human colorectal cancers. Nature. 1987;327:293-7.

9. Samuels Y, Wang Z, Bardelli A, Silliman N, Ptak J, Szabo S, Yan H, Gazdar A Powell SM, Riggins GJ, Willson JK, Markowitz S, Kinzler KW, Vogelstein B, Velculescu VE. High frequency of mutations of the PIK3CA gene in human cancers. Science. 2004:304:554.

10. Nassif NT, Lobo GP, Wu X, Henderson CJ, Morrison CD, Eng C, Jalaludin B, Segelov E. PTEN mutations are common in sporadic microsatellite stable colorectal cancer. Oncogene. 2004;23:617-28.

11. Greenhough A, Smartt HJ, Moore AE, Roberts HR, Williams AC, Paraskeva C, Kaidi A. The COX-2/PGE2 pathway: key roles in the hallmarks of cancer and adaptation to the tumour microenvironment. Carcinogenesis. 2009:30:377-86

12. Markowitz SD. Aspirin and colon cancer - targeting prevention? N Engl J Med. 2007:356:2195-8

13. Cha Yl, DuBois RN. NSAIDs and cancer prevention: targets downstream of COX-2. Annu Rev Med. 2007:58:239-52.

14. Atkin WS, Valori R, Kuipers EJ, et al. European guidelines for quality assurance in colorectal cancer screening and diagnosis. First edition-Colonoscopic surveillance following adenoma removal. Endoscopy. 2012; 44(Suppl 3):SE151-63.

15. Gerbe F, van Es JH, Makrini L, Brulin B, Mellitzer G, Robine S, Romagnolo B, Shroyer NF, Bourgaux JF, Pignodel C, Clevers $H$, Jay $P$. Distinct $\mathrm{ATOH} 1$ and Neurog3 requirements define tuft cells as a new secretory cell type in the intestinal epithelium. J Cell Biol. 2011;192: 767-80.

16. Jensen TSR, Mahmood B, Damm MB, Backe MB, Dahllöf MS, Poulsen SS, Hansen MB, Bindslev N. Combined activity of COX-1 and COX-2 is increased in non-neoplastic colonic mucosa from colorectal neoplasia patients. BMC Gastroenterol. 2018;18(1):31.

17. Courtney KD, Corcoran RB, Engelman JA. The PI3K pathway as drug target in human cancer. J Clin Oncol. 2010:28:1075-83.

18. Schubbert S, Shannon K, Bollag G. Hyperactive Ras in developmental disorders and cancer. Nat Rev Cancer. 2007;7:295-308.

19. Ying H, Kimmelman AC, Lyssiotis CA, Hua S, Chu GC, Fletcher-Sananikone E, et al. Oncogenic Kras maintains pancreatic tumors through regulation of anabolic glucose metabolism. Cell. 2012;149:656-70.

20. Chambard JC, Lefloch R, Pouyssegur J, Lenormand P. ERK implication in cell cycle regulation. Biochem Biophys Acta. 2007;1773:1299-310.

21. Rasola A, Sciacovelli M, Chiara F, Pantic B, Brusilow WS, Bernardi P. Activation of mitochondrial ERK protects cancer cells from death through inhibition of the permeability transition. Proc Natl Acad Sci U S A. 2010;107: 726-31.

22. Duhamel S, Hebert J, Gaboury L, Bouchard A, Simon R, Sauter G, et al. Sef downregulation by Ras causes MEK1/2 to become aberrantly nuclear localized leading to polyploidy and neoplastic transformation. Cancer Res. 2012:72:626-35.

23. Karnoub AE, Weinberg RA. Ras oncogenes: split personalities. Nat Rev Mol Cell Biol. 2008:9:517-31.

24. Deschênes-Simard X, Kottakis F, Meloche S, Ferbeyre G. ERKs in cancer: friends or foes? Cancer Res. 2014;74:412-9.

25. Deschenes-Simard X, Gaumont-Leclerc MF, Bourdeau V, Lessard F, Moiseeva O, Forest $V$, et al. Tumor suppressor activity of the ERK/MAPK pathway by promoting selective protein degradation. Genes Dev. 2013; 27:900-15.

26. Bric A, Miething C, Bialucha CU, Scuoppo C, Zender L, Krasnitz A, et al. Functional identification of tumor-suppressor genes through an in vivo RNA interference screen in a mouse lymphoma model. Cancer Cell. 2009;16:324-35

27. Eichhorn PJ, Creyghton MP, Bernards R. Protein phosphatase 2A regulatory subunits and cancer. Biochim Biophys Acta. 2009;1795:1-15.

28. Seshacharyulu P, Pandey P, Datta K, Batra SK. Phosphatase: PP2A structura importance, regulation and its aberrant expression in cancer. Cancer Lett. 2013;355:9-18.

29. Ghosh M, Tucker DE, Burchett SA, Leslie CC. Properties of the group IV phospholipase a (2) family. Prog Lipid Res. 2006:45:487-510.

30. Chandrasekharan JA, Sharma-Walia N. Lipoxins: nature's way to resolve inflammation. J Inflamm Res. 2015;8:181-92.

31. Kaltoft $N$, Tilotta MC, Witte $A B$, et al. Prostaglandin E2-induced colonic secretion in patients with and without colorectal neoplasia. BMC Gastroenterol. 2010;10:9. https://doi.org/10.1186/1471-230X-10-9.

32. Krishnan K, Ruffin MT, Normolle D, Shureiqi I, Burney K, Bailey J, Peters-Golden M, Rock CL, Boland CR, Brenner DE. Colonic mucosal prostaglandin E2 and cyclooxygenase expression before and after low aspirin doses in subjects at high risk or at normal risk for colorectal cancer. Cancer Epidemiol Biomark Prev. 2001;10:447-53.

33. Shoji $Y$, Takahashi M, Kitamura T, Watanabe K, Kawamori T, Maruyama T, et al. Downregulation of prostaglandin E receptor subtype EP3 during colon cancer development. Gut. 2004:53:1151-8.

34. O'Callaghan G, Houston A. Prostaglandin E2 and the EP receptors in malignancy: possible therapeutic targets? Br J Pharmacol. 2015;172: 5239-50. 
35. Woodward DF, Jones RL, Narumiya S. International union of basic and clinical pharmacology. LXXXIII: classification of prostanoid receptors, updating 15 years of progress. Pharmacol Rev. 2011;63:471-538.

36. Sonoshita M, Takaku K, Sasaki N, Sugimoto Y, Ushikubi F, Narumiya S, et al. Acceleration of intestinal polyposis through prostaglandin receptor EP2 in Apc (Delta 716) knockout mice. Nat Med. 2001;7:1048-51.

37. Holla VR, Backlund MG, Yang P, Newman RA, DuBois RN. Regulation of prostaglandin transporters in colorectal neoplasia. Cancer Prev Res. 2008;1:93-9.

38. Kleberg $\mathrm{K}$, Jensen $\mathrm{GM}$, Christensen DP, et al. Transporter function and cyclic AMP turnover in normal colonic mucosa from patients with and without colorectal neoplasia. BMC Gastroenterol. 2012;12:78. https://doi.org/10.1186/ 1471-230X-12-78.

39. Wang D, Dubois RN. Eicosanoids and cancer. Nat Rev Cancer. 2010;10:181-93.

\section{Publisher's Note}

Springer Nature remains neutral with regard to jurisdictional claims in published maps and institutional affiliations.

Ready to submit your research? Choose BMC and benefit from:

- fast, convenient online submission

- thorough peer review by experienced researchers in your field

- rapid publication on acceptance

- support for research data, including large and complex data types

- gold Open Access which fosters wider collaboration and increased citations

- maximum visibility for your research: over $100 \mathrm{M}$ website views per year

At BMC, research is always in progress.

Learn more biomedcentral.com/submissions 